\title{
A CLINICAL STUDY OF PRIMARY ANGLE CLOSURE GLAUCOMA AND ITS MANAGEMENT WITH Nd.YAG LASER IRIDOTOMY
}

\author{
Rajashree Reddy' ${ }^{1}$, M. R. Pujari ${ }^{2}$, Keerthi Duggineni ${ }^{3}$, Vishwanath Reddy ${ }^{4}$, Santosh Patil5, Prajwalli R6, Manjula Mangane ${ }^{7}$, \\ Kavita Salagar ${ }^{8}$
}

\begin{abstract}
${ }^{1}$ Associate Professor, Department of Ophthalmology, Mahadevappa Rampure Medical College. ${ }^{2}$ HOD, Department of Ophthalmology, Mahadevappa Rampure Medical College.

${ }^{3}$ Post Graduate, Department of Ophthalmology, Mahadevappa Rampure Medical College.

${ }^{4}$ Professor, Department of Ophthalmology, Mahadevappa Rampure Medical College.

${ }_{5}^{5}$ Associate Professor, Department of Ophthalmology, Mahadevappa Rampure Medical College.

${ }^{6}$ Assistant Professor, Department of Ophthalmology, Mahadevappa Rampure Medical College.

${ }^{7}$ Assistant Professor, Department of Ophthalmology, Mahadevappa Rampure Medical College.

${ }^{8}$ Asisstant Professor, Department of Ophthalmology, Mahadevappa Rampure Medical College.
\end{abstract}

\section{ABSTRACT}

\section{BACKGROUND}

Sixty million people are affected with glaucoma worldwide and more than 20 million have PACG. Of these, more than 5 million with PACG are blind, which is twice more than POAG. Early detection and timely treatment with Nd.YAG laser iridotomy and associated complications determine visual outcome.

\section{OBJECTIVES}

To study efficacy of Nd.YAG laser iridotomy in controlling intraocular pressure in primary angle closure glaucoma patients. To study role of prophylactic Nd.YAG laser iridotomy in the fellow eyes of primary angle closure glaucoma patients. To study anatomical changes in the angle of anterior chamber following peripheral iridotomy and complications of Nd.YAG laser iridotomy.

\section{METHODS}

A prospective study of 100 cases of primary angle closure glaucoma was conducted in Basaveshwar Teaching and General Hospital (Attached to M.R. Medical College), Kalaburagi. A detailed ophthalmic examination was performed. IOP was measured by Goldmann applanation tonometry. Gonioscopy was done by Goldmann 3 mirror lens. Nd.YAG laser was performed on all affected eyes and 82 fellow eyes of 100 patients and followed up for six months.

\section{RESULTS}

Among the patients included in the study 73 (73\%) patients were females and $27(27 \%)$ were males. In our study most of the patients were 40 to 60 years of age group. Our study included 57 (57\%) with PAC, 28 (28\%) with PACG and 15 (15\%) PACS. There was improvement of 2 Shaffer's grades in 65\%, 1 Shaffer's grades in $25 \%$ of patients. In my study 53 (92.2\%) of 57 PAC (Acute and sub-acute) patients had improved with stable visual acuities and good control of IOP at followup visits; 20 (71.4\%) of 28 PACG (Chronic) patients had good control of IOP at followup visits with improvement of stable visual acuities. All the PACS eyes and the fellow eyes with prophylactic laser iridotomy were with good IOP control and visual acuities; 6 (3.4\%) eyes out of 172 eyes which underwent iridotomies were found closed at follow-up visits with shallow anterior chamber and narrow angles. Transient elevation of IOP was noted in $38(38 \%)$ patients and iris bleeding was noted in 19 (19\%) patients. In our study, 28.5\% eyes with PACG and 5.2\% eyes with primary angle closure were classified as failures in controlling IOP and maintaining stable or improvement in visual acuity.

\section{INTERPRETATION AND CONCLUSION}

Nd. YAG laser iridotomy has now become treatment of choice in angle closure glaucoma. Efficacy of iridotomy depends on IOP at presentation, presence or absence of PAS, stage of the disease and pre-existing glaucomatous optic neuropathy. Effective screening and early intervention may improve visual outcomes.

\section{KEYWORDS}

Angle Closure, Papillary Block, Glaucoma, Nd.Yag Laser, Iridotomy.

HOW TO CITE THIS ARTICLE: Reddy R, Pujari MR, Duggineni K, et al. A clinical study of primary angle closure glaucoma and its management with Nd. Yag laser iridotomy. J. Evolution Med. Dent. Sci. 2016;5(11):472-477, DOI: 10.14260/jemds/2016/108

Financial or Other, Competing Interest: None.

Submission 04-11-2015, Peer Review 16-01-2016,

Acceptance 23-01-2016, Published 08-02-2016.

Corresponding Author:

Dr. Rajashree Reddy,

1-5-599/5, Balaji Colony, Tirupati-517501,

Andhra Pradesh

E-mail: riya3duggineni@gmail.com

DOI:10.14260/jemds/2016/108

\section{INTRODUCTION}

Glaucoma is a chronic progressive multifactorial optic neuropathy caused by a group of ocular conditions, which damage the optic nerve with a resultant loss of visual function.(1)

The most important risk factor is a raised intraocular pressure; 60 million people are affected with glaucoma worldwide and more than 20 million have PACG. Of these, more than 5 million with PACG are blind which is twice more 
than POAG.(2) Asians are most affected by PACG, while in the western world open angle glaucoma is more prevalent. (3)

It is estimated that by the year 2020 , there would be 79.6 millions people affected with glaucoma $47 \%$ of the glaucoma worldwide would be in Asia with more than $75 \%$ being angle closure.(4) At present 12 million Indians are affected by glaucoma accounting for $12.8 \%$ of the blindness in the country.

Hospital based data from India report POAG to be as common as PACG with $45 \%$ to $55 \%$ of primary glaucomas being PACG. $(5,6)$ In the glaucoma clinic of an eye hospital, $45.9 \%$ of all primary adult glaucomas were of angle closure type.(6)

Of these $24.8 \%$ had Acute Angle Closure Glaucoma (AACG), 31.2\% had subacute and $44 \%$ had chronic glaucoma. Since then the definition of angle closure has undergone change. Angle closure is now described as an anatomical disorder where symptomatology does not specify the involved mechanism.(7) In Asian eyes, the majority of the closures arise from a combination of a pupil block and nonpupil-blocking mechanisms.(8) Primary angle closure suspect (Occludable angle), Primary Angle Closure (PAC) and PACG are now distinct entities reflecting the severity of the disease. (3)

Primary angle closure glaucoma is a condition in which elevation of IOP occurs as a result of obstruction of aqueous outflow by partial or complete closure of the angle by peripheral iris. The diagnosis depends largely on examination of anterior segment and careful gonioscopy. It is the commonest type of glaucoma in Indian population.

Laser iridotomy has been shown to be an effective primary therapy for early PACG, although advanced cases require further treatment with topical medication or filtration surgery. Unaffected fellow eyes of patients who have had angle closure have a 50\% risk of developing an episode of angle closure if not treated with prophylactic iridotomy. Iridotomy acts by eliminating relative pupil block, which is the mechanism underlying the development of angle closure.

Keeping these aspects in view, the present study aims to study primary angle closure glaucoma with respect to its presentation, management with Nd:YAG laser iridotomy and post laser complications.

\section{MATERIALS AND METHODS}

The present study was conducted at Basaveshwar Teaching and General Hospital (BTGH) attached to M. R. Medical College, Gulbarga between December 2013 and May 2015. Approval and clearance was obtained from the Ethical Review Committee of the Institute.

A detailed history was elicited from the patient regarding onset, duration and progression and aggravating, relieving factors associated with symptoms. History of treatment taken previously, if any, for the same symptoms was noted. History of previous ocular problems, ocular trauma/surgery were enquired.

\section{Selection Criteria for the Patients}

1. Increase in the intraocular pressure in the primary angle closure glaucomas.

2. Patients with occludable angles as a measure of prophylaxis to prevent acute angles.

\section{Diagnostic Criteria}

An occludable angle was defined as one in which three quarters of the posterior trabecular meshwork was not visible on viewing with the Goldmann goniolens in the primary position of gaze.

Primary Angle Closure Glaucoma (PACG chronic) was diagnosed in eyes with occludable angles, PAS (Peripheral Anterior Synechiae) and glaucomatous optic neuropathy.

Evidence of glaucomatous optic neuropathy was defined as cup:disc ratio CDR of 0.7 or $0.2 \mathrm{CDR}$ asymmetry. In cases of early structural damage (0.7-0.8 CDR) or 0.2 asymmetry, a reproducible visual field defect is required to confirm the diagnosis.

Primary angle closure was diagnosed in eyes with an occludable angle, normal optic disc and visual field and any of the following:raised IOP ( $>19 \mathrm{mmHg}$ ), PAS, Pigment smearing in the superior angle or sequelae of the acute closure of angle (Eg: Glaukomflecken).

\section{Material Used}

Snellen's visual acuity was measured on all subjects. A slit lamp examination was carried out on every subject, looking for ischaemic sequelae of angle closure and signs of secondary glaucoma. The laser iridotomy site was examined for the patency by retro-illumination and direct visualization of the structures in the posterior pole. IOP was measured with a Goldmann Applanation tonometer.

Gonioscopy was performed with Goldmann gonioscopy lens. The width of iridotrabecular recess was recorded in all four quadrants. The angle was graded as occludable or open.

Dynamic (Indentation) gonioscopy using Zeiss fourmirror gonioscopy lens was performed to assess the presence or absence of PAS in each quadrant. Visual fields were assessed using static Humphrey's automated field analyzer.

\section{PROCEDURE}

1. Patients were explained in detail about the nature of the disease and the importance of timely intervention. Those with advanced disease were informed regarding the possible need for additional surgical intervention. The willingness of the patients for further follow-up visits was ensued prior to enrollment in study. The benefits and complications of Nd.YAG laser iridotomy were clearly explained to the patients.

2. An informed consent was taken.

3. Pupillary miosis: Pilocarpine nitrate $2 \%$ one drop every fifteen minutes beginning two hours before the procedure. Pupillary constriction by intense pretreatment with miotic like pilocarpine nitrate $2 \%$ topically causes maximum stretching and thinning of iris. It facilitates easy penetration or perforation of iris tissue by laser.

4. Control of IOP:

a. Pupillary miosis with Pilocarpine nitrate $2 \%$ also causes reduction in IOP; $1 \%$ Apraclonidine or 0.15 $0.2 \%$ Brimonidine tartrate eye drop 1 drop one hour before laser session effectively prevents post laser pressure spike also.

b. In glaucomatous eyes additionally carbonic anhydrase inhibitors (Acetazolamide $250 \mathrm{mg}$ ) one or two tablets should be given orally thirty minutes before the procedure. 
5. Improvement of corneal clarity:

a. Topical glycerol if available is used.

b. Sodium chloride $5 \%$ eye drops may be alternatively used topically to reduce corneal edema- 1 drop every 15 minutes beginning one hour before the procedure.

6. Anaesthesia: Topical lignocaine $4 \%$ or preferably proparacaine HCL $0.5 \%$ eye drop is used before insertion of contact lens.

7. Comfortable positioning of patient.

8. Steady fixation obtained by head strap and adjusting fixation light.

9. Insertion of contact lens: Commonly Abraham contact lens was used, to which methylcellulose is added for patient comfort and is placed on the patient's cornea.

10. Illumination of laser room: Darkened/semi-darkened.

11. Iridotomy technique proper.

\section{In phakic eye}

- Usually 3-8 mJ per shot from Q switched Nd:YAG laser and one to three shots were sufficient to complete the procedure.

- If the anterior chamber depth is nil, the pulse energy should not exceed 10-12 mJ.

- Burst mode is not performed since hole in iris may occur before completion of burst leading to damage of anterior lens capsule.

\section{Optimal site of iridotomy}

a. Between 10 o'clock and 2 o'clock, so that it is covered by eyelid.

b. Avoid 12 o'clock site, here gas bubble may obstruct the site of the opening.

c. About three-fourth of the distance between pupillary margin and iris periphery.

d. Avoid arcus senilis as its opacity interferes with the clear focus and transmits less laser energy.

- Usually laser shots are aimed at periphery of iris onto iris crypts or area of thin stroma, where iris thickness is less. This also avoids injury to anterior lens capsule due to peripheral location. Lens is curved posteriorly towards periphery/equator. Thus, the peripheral part of anterior lens capsule is not adjacent to posterior surface of peripheral iris.

- If an iridotomy opening is too small, we should try to create additional Nd:YAG iridotomy at a separate site (Nd:YAG laser should not be used to enlarge a small iridotomy opening to avoid injury to anterior lens capsule).

- $\quad$ End point in perforation of iris (Iridotomy) is identified by

a. Sudden outpouring of melanotic pigments from posterior chamber to anterior chamber.

b. Sudden deepening of anterior chamber.

c. Sudden shower of pigments in pupillary area.

Nd:YAG laser iridotomy was done on 182 eyes of 100 patients. Average period of followup was 6 months.

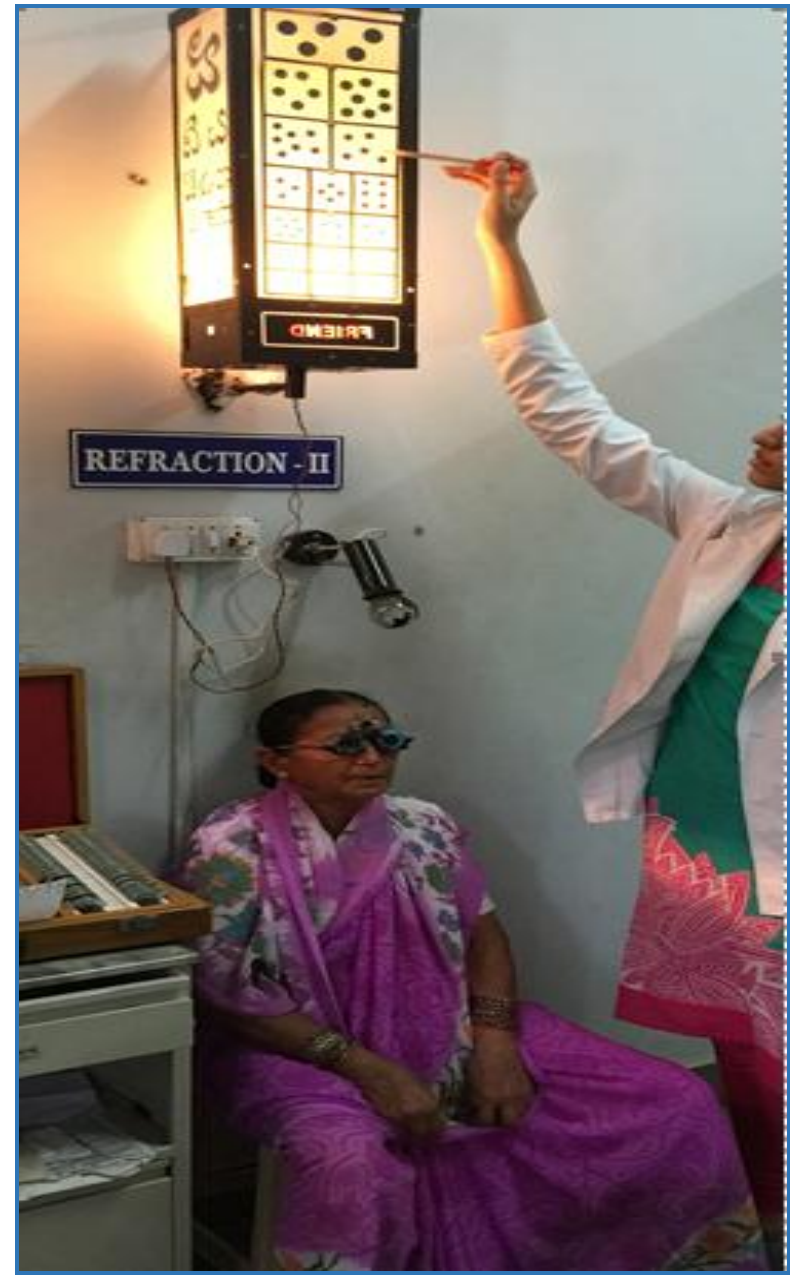

Fig. 1: Testing of Visual Acuity

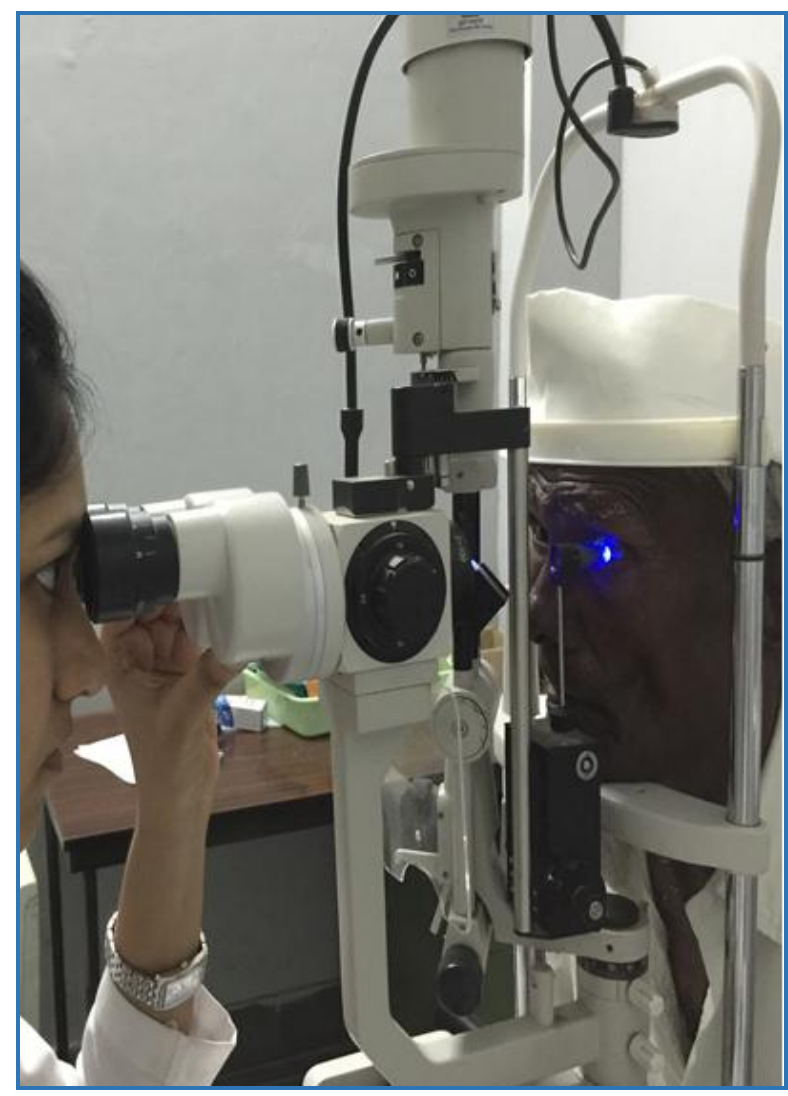

Fig. 2: Applanation Tonometry 


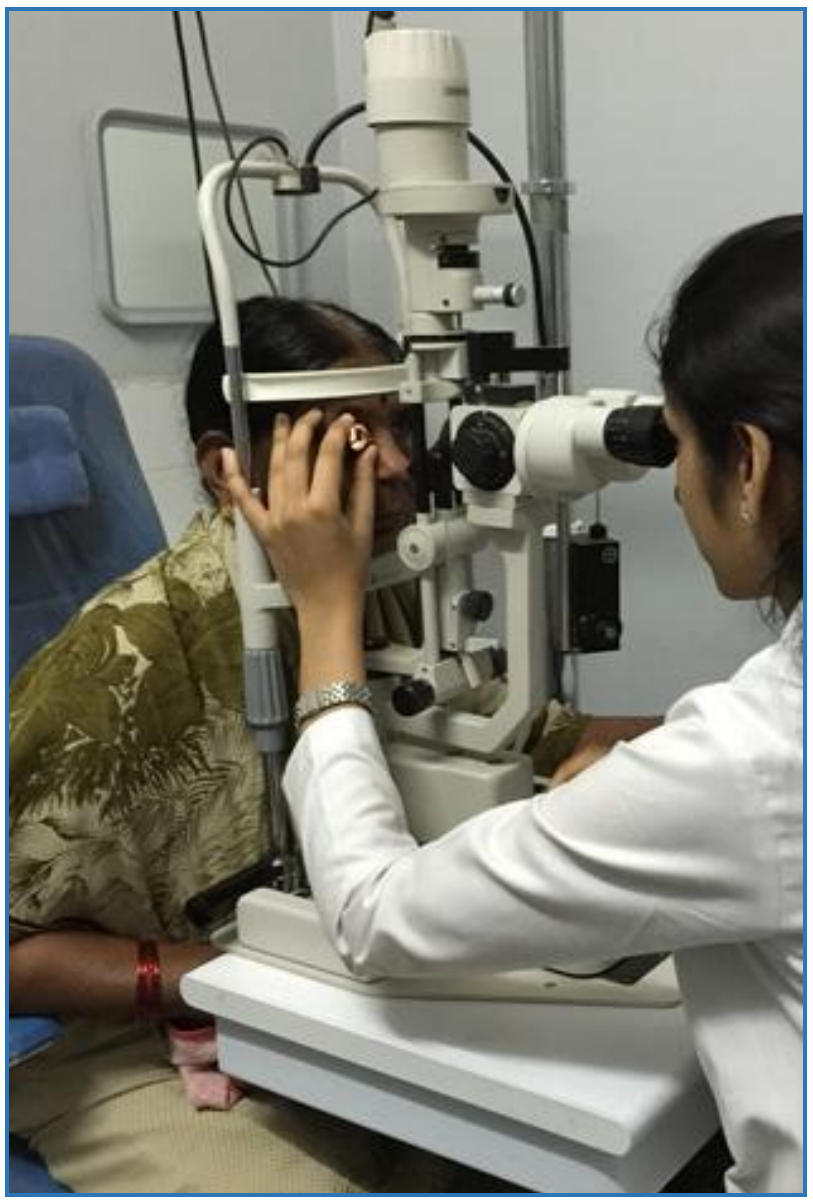

Fig. 3: Gonioscopy

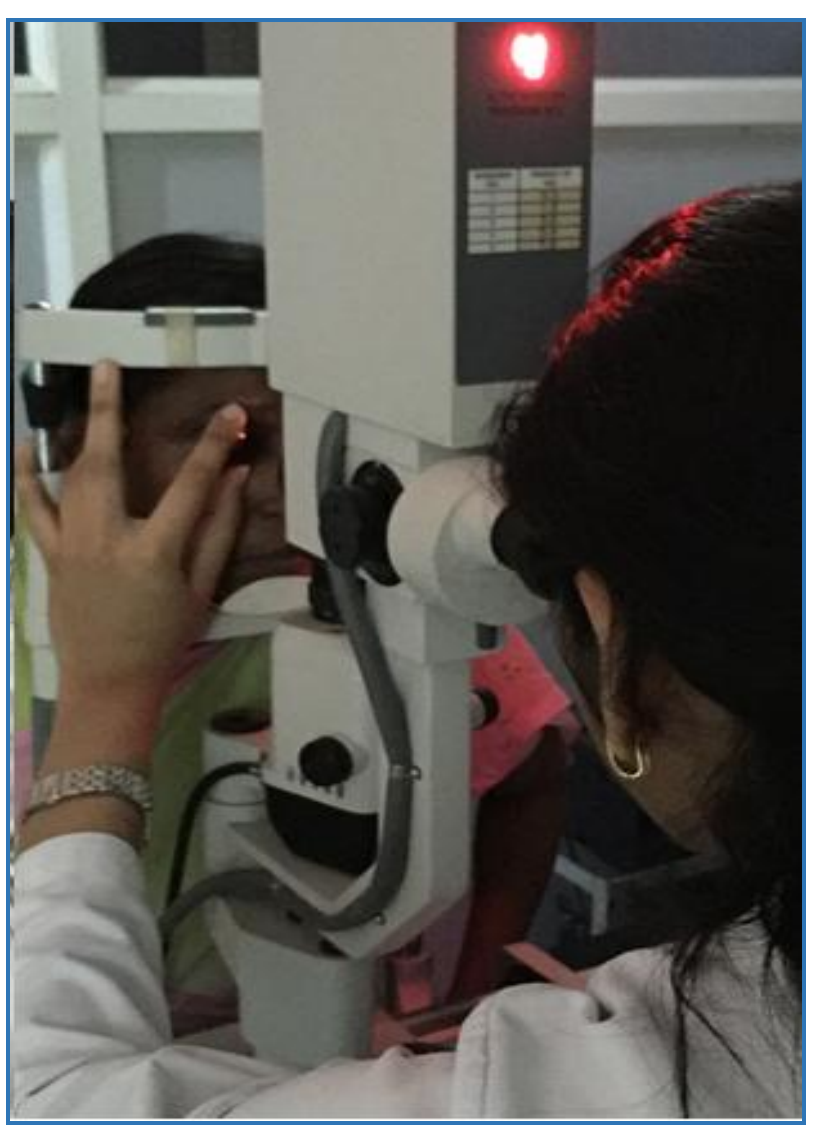

Fig. 4: Nd. YAG Laser Iridotomy
RESULTS

\begin{tabular}{|c|c|c|c|}
\hline Age (Years) & Male & Female & Total \\
\hline $31-40$ & 0 & 4 & 4 \\
\hline $41-50$ & 10 & 29 & 39 \\
\hline $51-60$ & 14 & 23 & 37 \\
\hline $61-70$ & 3 & 17 & 20 \\
\hline Table 3: Age and sex distributions of study populations \\
\hline
\end{tabular}

Highest group of patients in my study were between 41-50 years (39). Second highest incidence was between 51-60 yrs.

\begin{tabular}{|c|c|}
\hline Sex & Number of Cases \\
\hline Male & 27 \\
\hline Female & 73 \\
\hline \multicolumn{2}{|c|}{ Table 4: Sex Distribution } \\
\hline
\end{tabular}

In my study, female patients (73 out of 100) are more in number.

\begin{tabular}{|c|c|}
\hline Diagnosis & Number of Cases \\
\hline $\begin{array}{l}\text { PACG-Primary Angle Closure } \\
\text { Glaucoma (Chronic) }\end{array}$ & 28 \\
\hline $\begin{array}{c}\text { PAC-Primary Angle Closure (Acute } \\
\text { and Subacute) }\end{array}$ & 57 \\
\hline $\begin{array}{c}\text { PACS-Primary Angle Closure } \\
\text { Suspects }\end{array}$ & 15 \\
\hline Total & 100 \\
\hline \multicolumn{2}{|c|}{$\begin{array}{c}\text { Table 5: Initial diagnosis in eyes treated with } Y A G \\
\text { laser iridotomy }\end{array}$} \\
\hline
\end{tabular}

\begin{tabular}{|c|c|c|}
\hline PACG (Chronic) & $\begin{array}{c}\text { PAC } \\
\text { (Acute and Subacute) }\end{array}$ & PACS \\
\hline $8(28)$ & $3(57)$ & 0 \\
\hline \multicolumn{3}{|c|}{ Table 6: Failures of YAG PI } \\
\hline
\end{tabular}

\begin{tabular}{|c|c|c|}
\hline Complication & $\begin{array}{c}\text { Number of } \\
\text { Cases }\end{array}$ & Percentage \\
\hline $\begin{array}{c}\text { Transient elevation of } \\
\text { IOP (>8mm) }\end{array}$ & 38 & 38 \\
\hline Aqueous flare/debris & 34 & 34 \\
\hline Bleeding of iris & 19 & 19 \\
\hline Corneal burns & 5 & 5 \\
\hline Lens damage & 4 & 4 \\
\hline \multicolumn{2}{|c|}{ Table 7: Complications of YAG Iridotomy }
\end{tabular}

\section{DISCUSSION}

Nd-YAG laser iridotomy for the primary angle closure glaucoma was performed at Basaveshwar Teaching and General Hospital during the period of December 2013 to May 2015.

Subjects with complete synechial angle closure and with glaucomatous optic atrophy excluded from the study group.

All stages of glaucoma were treated and followed up during the study period. Average period of followup was 6 months. All glaucoma suspects were evaluated further at Glaucoma Clinic, Basaveshwar Teaching and General Hospital.

Among the patients included in the study 73 (73\%) patients were females and $27(27 \%)$ were males indicating a 3:1 ratio of female:male preponderance.

In our study, most of the patients were 40 to 60 years of age group. Studies from European community prove 
incidence of angle closure is more between 50-70 years group. Thus angle closure glaucoma occurs a decade earlier in this population study.

Our study included 57 (57\%) with primary angle closure (Acute and sub-acute), 28 (28\%) patients with primary angle closure with glaucomatous optic neuropathy, and $15(15 \%)$ primary angle closure suspects.

Average amount of laser energy required for making laser iridotomy was $10 \mathrm{~mJ}$. An average of 3 shots were required.

\section{Gonioscopy}

There was a definite improvement of 1 to 2 Shaffer's grades of angle of anterior chamber. There was improvement of 2 Shaffer's grades in $65 \%$ of patients. Improvement of one Shaffer's grade was noted in $25 \%$ of patients and there was little or no improvement in Shaffer's grades in $10 \%$ of eyes with PAS in 3 quadrants of anterior chamber angle.

\section{Intraocular Pressure}

In my study $53(92.2 \%)$ of 57 PAC (Acute and sub-acute) patients had improved with stable visual acuities and good control of IOP at followup visits; 20 (71.4\%) of 28 PACG (Chronic) patients had good control of IOP at followup visits with improvement of stable visual acuties. All the PACS eyes and the fellow eyes with prophylactic laser iridotomy were with good IOP control and visual acuities. None of the PAC and fellow eyes suffered symptomatic angle closure or glaucomatous optic neuropathy in followup period.

8 (28.5\%) patients of PACG group (Chronic) were presented with uncontrolled IOP at followup visits. Out of 8 of chronic angle closure group, 5 patients had PAS in more than 3 quadrants of angle structures and 3 had PACS in more than 2 quadrants on followup gonioscopy. These were treated with filtration surgeries; 4 of them underwent trabeculectomy and 3 of them underwent combined procedures who are associated with immature cataract. One patient with good compliance and apprehensive for surgery had been maintained on long term medication with good control of IOP and stable visual fields.

Three of the primary angle closure (Acute and subacute), group who had uncontrolled IOP were advised filtration surgeries; 2 of them were associated with synechial angle closure and 1 associated with pseudoexfoliation; 2 of them underwent trabeculectomy 1 with combined procedure with good control IOP at followup visits.

\section{Patency of Yag Iridotomy Hole}

The size of iridotomy hole is not smaller than $0.2 \mathrm{~mm} 2.6$ (3.4\%) eyes out of 172 eyes, which underwent iridotomies were found closed at followup visits with shallow anterior chamber and narrow angles; 10 eyes which underwent filtration surgeries excluded. Most of them found occluded within a period of 4-8 weeks of followup. Second time iridotomy was performed at another site with appropriate size hole. It had been recorded that most of these cases had small iridotomy holes at the time of treatment or with intense aqueous flare and tissue reaction after iridotomy. Second time iridotomy was good enough and found patent in all 6 eyes at followup.

\section{Prophylactic Iridotomy}

YAG PI was effective in all the fellow eyes of PACG and PAC and prophylactic iridotomies of PACS eyes. None of the PACS and fellow eyes of PACG and PAC suffered symptomatic angle closure or glaucomatous optic neuropathy.

\section{Complications of YAG $P$}

In our study, transient elevation of IOP was noted in 38 (38\%) patients and iris bleeding was noted in 19 (19\%) patients. These complications were attributed to the increased amount of laser energy required for dark brown Indian iris.

Other complications like corneal burns, aqueous flare, pigment dispersion were mild. Corneal burns were treated with pad and bandage. Mild dose topical steroids (fluorometholone) were used for mild iritis for a period of 3-4 days.

\section{Failure of YAG PI}

In a study on primary angle closure in East Asian eyes by Nolan et al. $47 \%$ eyes with glaucomatous optic atrophy and $4 \%$ eyes with primary angle closure were classified as failures and were required further filtration surgeries.

In our study $28.5 \%$ eyes with PACG and 5.2\% eyes with primary angle closure were classified as failures in controlling IOP and maintaining stable or improvement in visual acuity. These further required filtration surgeries.

\section{CONCLUSION}

Nd.YAG laser iridotomy was performed over 182 eyes of 100 patients. Among them 28 were PACG (Chronic), 57 PAC (Acute and subacute) and 15 PACS (Suspects) patients.

All these patients were treated with Nd.YAG laser iridotomy after controlling initial presenting intraocular pressure and preparing the patient for the procedure by instilling topical miotic and anaesthetic eye drops.

Female patients were 3 times more commoner than male patients. Most of the patients were in their fifth to sixth decades.

An average of $10 \mathrm{~mJ}$ was required to treat these dark Indian iris. There was definite improvement of anatomy of angle structures by about one or two Shaffer's grades.

IOP was well controlled in 71.4\% (20) patients of PACG (Chronic) and 92.2\% (53) of PAC (Acute and subacute) group. Most of the uncontrolled group presented with synechial angle closure at followup visits and were treated with filtration surgeries and a very few with long term medication.

All the PACS (suspects) group and fellow eyes of PAC and PACS groups were treated with prophylactic YAG laser iridotomy. None of them suffered symptomatic angle closure or glaucomatous optic neuropathy during followup visits.

There were very minor complications like transient elevation of IOP in 38\% of patients and iris bleeding in 19\% of patients after iridotomy. These were treated effectively after iridotomy. Other minor complications included corneal burns, aqueous flare and pigment dispersion.

The outcome of the cases in this study was related to the stage of the disease at diagnosis. Around twenty eight percent of eyes with PACG were classed as treatment failures on grounds that IOP was poorly controlled or visual acuity had deteriorated due to progression of glaucoma; $5.2 \%$ of PAC eyes were found to have glaucomatous optic neuropathy 
at followup, but there were other features such as pseudoexfoliation to explain a poor prognosis. None of the PACS eyes suffered symptomatic angle closure or glaucomatous optic neuropathy. It appears that once extensive synechial angle closure and glaucomatous optic neuropathy have developed, an iridotomy will be less likely to reduce IOP and protect visual function than in eyes treated at an earlier stage.

In my study Nd.YAG laser iridotomy proved to be effective in widening the drainage angle and reducing elevated IOP in primary angle closure patients. This suggested that pupil block is a significant mechanism causing closure of the angle in this population. Nd.YAG laser iridotomy is a safe and non-invasive procedure.

\section{BIBLIOGRAPHY}

1. Sihota R. The glaucomas. Parsons' diseases of the eye. New Delhi: 20th Edition. Elsevier; 2007.
2. Becker-Shaffer's Diagnosis and Therapy of the Glaucomas. 8th edition Pg Nos: 47.70,71,74,78,80,81.

3. Sood D, Sood NN. Angle closure and India. Indian J Ophthalmol. 2006;54(3):147-148.

4. Quigley HA, Broman AT. The number of people with glaucoma worldwide. Br J Ophthalmol 2006;90:262-7.

5. Ramakrishnan R, Nirmalan PK, Krishnadas R, et al. Glaucoma in a rural population in Southern India. Ophthalmology 2003;110:1484-90.

6. Sihota R, Agarwal HC. Profile of the subtypes of angle closure glaucoma in a tertiary hospital in North India. Indian J Ophthalmol 1998;46:25-9.

7. He M, Foster PJ, Johnson GJ, et al. Angle closure glaucoma in East Asian and European people. Different Diseases? Eye 2006;20:3-12.

8. Dandona R, Dandona L. Review of findings of the Andhra Pradesh Eye Disease Study: policy implications for eye care services. Ind J Ophthalmol 2001;49:215234. 